\title{
A NOVEL APPLICATION OF PRE-SIGNALS TO IMPROVE THE PERFORMANCE OF SIGNALIZED INTERSECTIONS: EVALUATION THROUGH SIMULATION
}

\author{
António A. C. Vieira ${ }^{(\mathrm{a})}$, Luís M. S. Dias ${ }^{(\mathrm{b})}$, Guilherme A. B. Pereira ${ }^{(\mathrm{c})}$, José A. Oliveira ${ }^{(\mathrm{d})}$ \\ (a),(b),(c),(d) University of Minho, 4710-057, Braga, Portugal \\ ALGORITMI Research Center \\ (a) $\underline{\text { antonio.vieira@dps.uminho.pt, }},{ }^{(\mathrm{b})} \underline{1 \mathrm{sd} @ \text { dps.uminho.pt, }}{ }^{(\mathrm{c})}$ gui@dps.uminho.pt,${ }^{(\mathrm{d})} \underline{\mathrm{zan} @ \mathrm{dps} . u m i n h o . p t}$
}

\begin{abstract}
To ponder less costlier solutions to solve traffic congestion problems at signalized intersections, this paper proposes a novel application consisting of using pre-signals. Hence, an agent-based traffic simulation model was developed, where it is possible to model different types of intersections - including roundabouts of different sizes - and quantify and compare their performance. By analyzing the simulation results, it was found that: on the intersection with pre-signals, an increase in the flow of $10 \%$ and $3 \%$ was registered, the vehicles spent 1 and 2 less minutes to cross the intersection and the fuel consumption was decreased in $22 \%$ and $44 \%$, in comparison to regular intersections and roundabouts, respectively. Concerning the size of queues, it was noted that the queues of the regular intersection were 60 meters longer than the queues on the intersection with pre-signals and on the roundabout. Based on these findings, and by making cost assumptions, a small cost analysis was made, which indicates that at least 1 million $€$ could be yearly saved.
\end{abstract}

Keywords: Discrete-Event Simulation, Agent Modelling, Signalized Intersection, Pre-signals, Roundabout.

\section{INTRODUCTION}

Traffic congestion problems are becoming increasingly intense, due to the growing number of vehicles circulating on the roads. Because of this, traffic engineers and academics have been focusing on ways to improve the capacity, and other performance indicators, of traffic intersections, since these are the most common bottlenecks of the traffic congestion problems. Many times, the solutions to these problems are onerous constructions, e.g.: tunnels or bridges.

The purpose of this paper is to propose a novel approach, consisting on using pre-signals on the approaches of a signalized intersection to improve its performance. To evaluate the proposed approach, the authors developed a discrete-event simulation model in Simio (A. Vieira et al. 2015; A. A. C. Vieira et al. 2018), a recent simulation tool that allows the user to build models employing agent-modelling concepts, which consists in individually modelling the behaviour and characteristics of each entity, i.e., each vehicle travelling through the model. Hence, in this paper, the authors will use the developed simulation model to quantify and compare the results obtained by the simulation model, in order to assess the proposed novel approach for pre-signals. In addition, a brief analysis of cost assumptions will be made.

Some of the obtained results have already been published in previous publications. Vieira et al. (2014) firstly compared the performance of regular signalized intersections and intersections applying the proposed pre-signals. The results indicated that both types of intersections performed better for different green light durations and thus a balance between gains and losses, when considering the duration of the green light to use on a regular traffic light intersection, needs to exist. In this type of intersections, durations around 40 to 60 seconds should be used, since increasing it too much would result in a high flow, but also on high waiting times per vehicle and queue sizes, which was also observed by Pan et al. (2010), since the time that the vehicles, on the remaining lanes, wait for the green signal also increases. On the other hand, if low durations of green signal are used, the capacity at a steady level without reducing queue lengths and waiting time. In this regard, it was concluded that the performance increased when low durations of green signal are used. Moreover, the results also showed that for low traffic congestion, the distance between presignals and the main traffic lights did not affect the performance of the intersection, while for high congestion scenarios the best performance was achieved for distances of more than 40 meters. In Vieira et al. (2017), the intersection with pre-signals was compared with roundabouts.

For this study, the SIMIO simulation software was used. It should be noted that, of the reviewed studies, none used discrete-event simulation to assess the performance of the proposed implementations. Several studies exist comparing discrete-event simulation tools, considering several factors (Dias et al. 2016; Oueida et al. 2016; António Vieira et al. 2014; V Hlupic and Paul 1999; Vlatka Hlupic 2000).

A pre-signal is an additional traffic signal located upstream of the main traffic signal. The use given to 
pre-signals found in literature and in practice can be divided in two sets: to increase the capacity of signalized intersections, by giving priority to the leftturn maneuvers, or to give priority to buses. One of the studies the can be fit in the former was proposed by Xuan et al. (2011). The authors proposed a strategy in which the area upstream to the pre-signal is used to separately queue vehicles wanting to make a left turn from the ones wanting to go straight. The pre-signals of these lanes alternatively give green time to these two sets of vehicles, allowing them to enter the area upstream to the main signal and use all lanes for these operations. According to the authors, this way, the capacity of the intersection can be improved. More recently, several studies have demonstrated benefits of this method, in terms of increased capacity, decreased delay and travel time, efficiency of land use, increased safety, and other aspects (Li et al. 2014; Yan, Jiang, and Xie 2014; Yang and Shi 2017; Zhou and Zhuang 2014; Cai et al. 2016).

A similar strategy to the one proposed by Xuan et al. (2011), consists in using an opposite lane to make a left turn, when the convergent flow is with red signal. In this strategy, the pre-signals work as pre-intersections, located at a certain distance upstream to the main intersection, since they regulate the vehicles that want to turn left and the ones that want to access the same approach through an opposing direction (Zhao et al. 2013, 2015; Zhao and Liu 2017; Wu et al. 2016; Kozey, Xuan, and Cassidy 2016; Zhao, Liu, and Di 2016).

One of the first uses given to pre-signals was to give priority to buses in multi lanes with an exclusive bus lane (Oakes, Hellmann, and .Kelly 1994). Whilst buses can advance to the area upstream to the main signal, through their exclusive lane, other vehicles are queued upstream to the pre-signal, thus giving priority to buses. Guler and Menendez (2014 b) empirically evaluate the use of pre-signals in intersections to give priority to buses. In this implementation of pre-signals, these automatically turn red when a bus arrives to the presignal, regardless of the main signal's phase, thus only allowing buses to be queued upstream to the main signal. The same authors (2014 a) also proposed a method to give priority to buses using pre-signals, in which car are alternatively queued in the zones upstream to the pre-signal and the main signal, until the arrival of a bus, which triggers the pre-signal's red phase, allowing the bus to have priority in the main intersection. In both studies, the authors used queuing theory, to quantify and empirically evaluate the delays encountered by cars and buses. With their studies, the authors wanted to demonstrate that their application of pre-signals could significantly reduce the total person hours of delays for both cars and buses.

Guler and Menendez (2016) have also presented an implementation for pre-signals in which these are used to give priority to buses in single-lane intersections, which do not have dedicated bus lanes. In their proposal, on each lane of an intersection, a pre-signal is located upstream and downstream of the main signal, wherein the pre-signal in the opposite lane is nearer to the intersection, than the pre-signal on the remaining lane. This way, buses can bypass vehicles queued on the pre-signal upstream to the intersection, through a bidirectional lane segment, formed as a result of the distance between the pre-signals and located in the lane of the opposite direction. The authors used analytical equations to assess their proposal.

According to Guler and Menendez (2014 b), there is a lack of empirical evaluation on the use of pre-signals, probably due to its scarse implementation across the world. Therefore, simulation could be used to evaluate these novel implementations. Moreover, it allows the modeler to make experiments on the computer, rather than on the field, allowing to thoroughly analyze the proposed pre-signals implementation (A. A. Vieira et al. 2018).

The analyzed researchers agree that these various applications of pre-signals improve the performance of the traffic operations in which they were applied to. In any case, the reviewed applications are either focused on giving priority to buses or improving the capacity of intersections with high left turn demand. However, the purpose of pre-signals proposed in this paper has a different application and purpose. In fact, to the best of the authors' knowledge, is the first time an application of this kind is proposed.

The pre-signals' implementation proposed in this paper is described in the next section. Third section gives a brief contextualization on how the simulation model was developed. The results will be discussed in the fourth section and conclusions, as well as some future work to better cement this new concept, will be addressed in the last section.

\section{OPERATION OF PRE-SIGNALS}

The pre-signals' implementation proposed in this paper consists on making the space upstream to the main intersection available for all vehicles to accelerate, from their rest state, before the main signal changes to green. To that end, vehicles are queued on the pre-signal and this traffic light changes to green before the main signal, to allow the queued vehicles to notice the change (reaction time) and initiate their start-up acceleration process. With this implementation of pre-signals, an intersection with continuous flow is expected to be achieved, since:

- The reaction time of drivers is eliminated, since when the pre-signal changes to green, the main one is still red;

- The impact of the start-up acceleration is reduced, since the vehicles initiate their startup acceleration process, while the main signal is still red;

- Whilst the main signal phase of an approaching is ending, the pre-signal of the next approach can change to green, allowing the first vehicle of the second approach to cross the intersection few seconds after the last 
of the first approach. This time lag between phases of different approaches should be analyzed for security reasons.

Figure 1 shows an example of the duration of all signals of both pre-signal and main traffic light, in two consecutive approaches of the same intersection. It should be noted that these durations were not used in the intersection modelled with pre-signals in the simulation model; they are just an example.

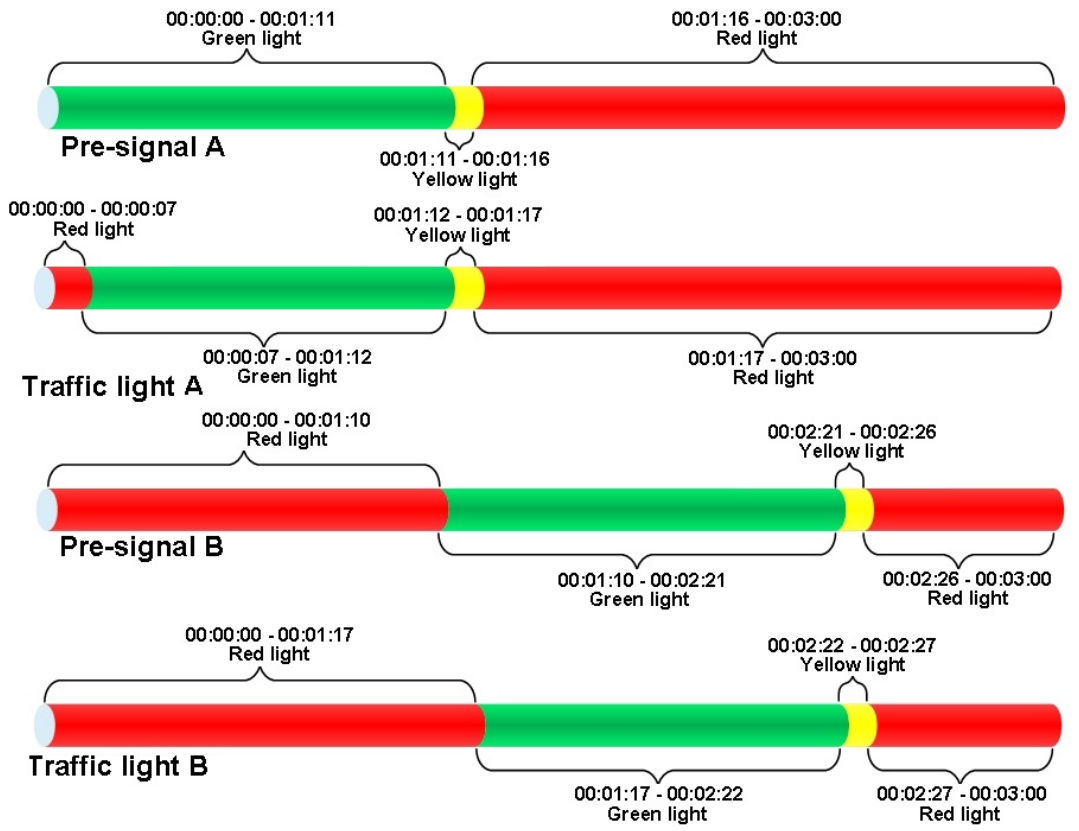

Figure 1: Example of the duration of signals in an intersection with pre-signals (A. Vieira et al. 2014).

The duration of the green phase of the pre-signals is equal to green main signal plus an additional time, before the main green signal and minus another interval duration, before the main green signal. In a case where the first interval is too high, vehicles would reach the main signal, still in red phase, and thus would need to slowdown, or even stop. On the other hand, this interval cannot be too low, otherwise vehicles would not have the sufficient time to reach the main signal with considerable speed, i.e., pre-signals would not serve its purpose of reducing the impact of the reaction time of drivers and start-up acceleration. Regarding the second interval, if it is too high, vehicles would be queued in the pre-signal, with many seconds of main green signal remaining, whereas too low values would result in queued vehicles in the main signal, which would affect the start-up acceleration process of vehicles from the next phase, since these would not have the required space to accelerate.

Besides this, there are also security concerns involved in the phases of the pre-signal. In other words, if its purpose is to allow vehicles from all approaches to cross the intersection at near-to-cruise speed and few seconds of interval between approaches, it still needs to ensure that no accidents occur. The duration of green signa of the pre-signal can be of particular importance in this regard.

\section{MODELING}

In Simio, the modeler can place and connect $3 \mathrm{~d}$ objects, which represent the real elements of the system in analysis. Thereafter, additional logic can be added, by using other simulation paradigms, e.g.: processes. Furthermore, concepts of agent modelling are also present in this software, allowing the user to model the individual behaviour of each entity. Thus, in this case, it is possible to have vehicles set their actions, e.g. accelerate and maintain a safety distance to the leading vehicle. Figure 2 shows the $2 \mathrm{D}$ representation of the simulation model. Each square represents a distance of 10 meters.

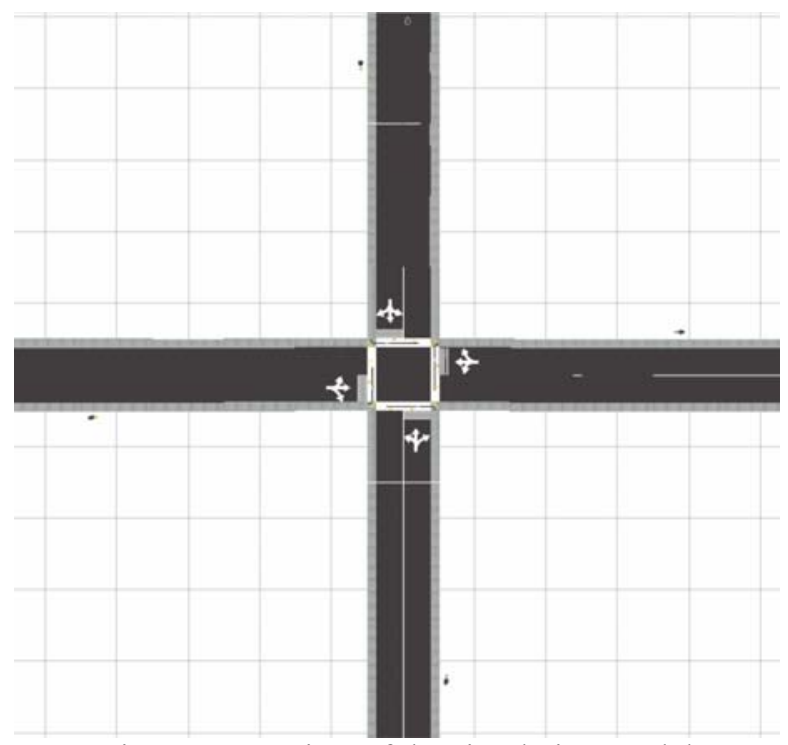

Figure 2: 2D view of the simulation model 
As can be seen, the signalized intersection has four approaches, each one with two lanes - one for inflow and another for outflow traffic on the intersection. When the vehicles enter the intersection, regardless of the approach where they are, they can choose the intersection through any exit.

In this simulation model, the traffic lights exist, although merely for animation purposes, since their locations are modelled as geographic coordinates and a data structure, which represents the state of each trafficlight. This way, the position of the signals, including pre-signals, can be easily edited. The signal cycles are processed on a counter clockwise direction, starting with green, changing to yellow and thereafter to red, before repeating the cycle. Only an approach at a time has green signal.

As soon as vehicles are created, they start executing a process that remains active until they cross the intersection, modelling their behaviour according to the situations through which they pass. Some of these processes and the input data used in these models, can be consulted with more detail in the previous publications of this project (A. Vieira et al. 2014; A. A. C. Vieira et al. 2017). Vehicles can even communicate between them to consult, for instance, the speed of the vehicle ahead, the distance to a given object (e.g. traffic-light, stopping line, etc.), or the flasher sign of a vehicle on a roundabout, among others. This modelling paradigm, or philosophy, is known as agent modelling, since entities are modelled as agents, each one being able to make decisions in an independent way, like accelerating or slowing down, considering their surroundings.

To model roundabouts, in Simio, it is possible to add models to a project, using the elements of other models of this project. Thus, in this case, a new model was added to this project, which uses most of the processes that already existed. Thereafter, we only needed to change the design of the intersection and model the rules to access the roundabout. This was of particular importance, since it allowed the modelers to use the same elements in all intersections that would later be compared. For instance, all vehicles in all intersections use the same processes to slowdown or accelerate. Figure 4 and Figure 4 respectively show the simulation models of signalized intersections and roundabouts during runtime.

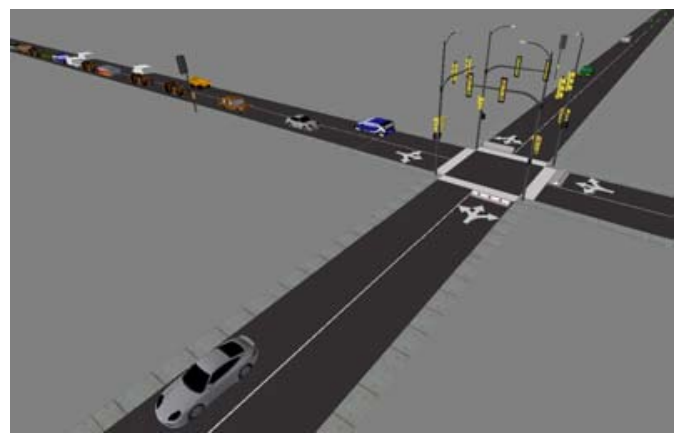

Figure 3: 3D view of the modelled signalized intersection.

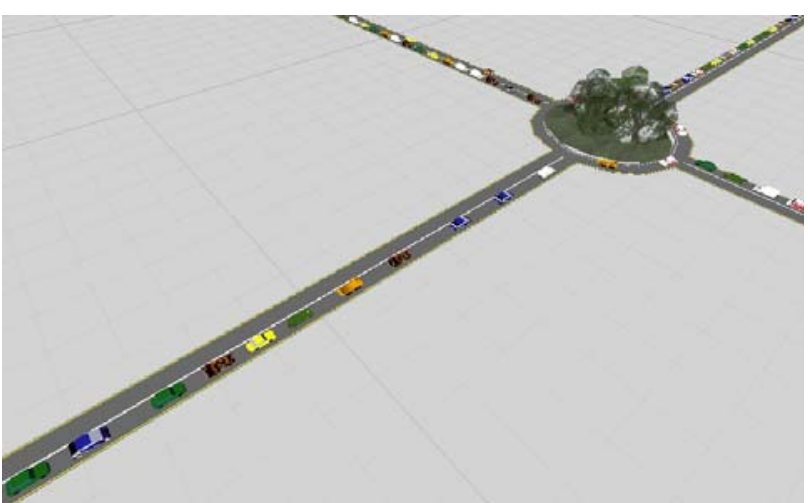

Figure 4: 3D view of the modelled roundabout.

\section{SIMULATION EXPERIMENTS}

In this section, the results obtained in the simulation model will be analysed. The following are parameters of the conducted simulation experiments: frequency with which the vehicles arrive to the system (traffic intensity) and type of intersection, i.e., roundabout or intersection with and without pre-signals. Moreover, the values 4, 8, 13 and 50 seconds were considered, respectively, for the traffic intensity, and hence the following respective levels were considered: very high, high, medium and low traffic. A warm-period of 360 seconds was used, along with a simulation time of 2 hours and 6 replications. As KPI (Key Performance Indicators), the following were defined:

- $\quad$ Average crossing time. This KPI is the elapsed time between the time when a vehicle is created and when it travels an additional distance of 150 meters after having crossed the intersection.

- Average flow of vehicles in vehicles/hour. This KPI is the inverse of the time interval between passages of vehicles through the intersection;

- Average number of vehicles on the queues. This KPI is measured every minute;

- Average flow of vehicles in vehicles/hour. This KPI is the inverse of the time interval between passages of vehicles through the intersection;

- Average total fuel consumed per vehicle in milligrams; and average total emissions of vehicles in milligrams ( $\mathrm{CO}, \mathrm{HC}$ and $\mathrm{NOx})$. These, start being accounted when vehicles are created and are updated every minute. When vehicle cross the intersection, these values are recorded;

- Average number of stops per vehicle.

In this section the performance of each intersection will be analysed in an average day and year perspective. The obtained results can be consulted in Table 1, 
Table 2 and Table 3. The displayed values were obtained, considering the formulae given below, in which KPI stands for Key Performance Indicators, $\theta$ refers to a number of hours in a traffic intensity and $\alpha$ represents a type of intersection. It should be noted that by using these formulae, the intention is not to exactly determine the respective value, but rather to have an estimation of the what could be the magnitude order of those values, by applying the formulae equally to all analyzed types of intersections. Thus, there is certainly an uncertainty degree associated to these formulae.

\section{Total flow of vehicles during an average day
Total time to cross the intersection during an average day \\ Total fuel consumed during an average day \\ $K P L_{\gamma}=\{$ Total CO emissions during an average day Total HC emissions during an average day Total NOx emissions during an average day Space occupied by a queue during an average day}

$\theta_{t}=\left\{\begin{array}{l}\text { Hours of Very High Traf fic Intensity } \\ \text { Hours of High Traffic Intensity } \\ \text { Hours of Medium Traffic Intensity } \\ \text { Hours of Low Traffic Intensity }\end{array}\right.$

$i \in\left\{\begin{array}{l}\text { Very High Traffic Intensity } \\ \text { High Traf fic Intensity } \\ \text { Medium Traf fic Intensity } \\ \text { Low Traffic Intensity }\end{array}\right.$

$$
\begin{aligned}
& \text { if } \gamma=1 \\
& \text { if } \gamma=2 \\
& \text { if } \gamma=3 \\
& \text { if } \gamma=4 \\
& \text { if } \gamma=5 \\
& \text { if } \gamma=6 \\
& \text { if } \gamma=7 \\
& \text { if } i=1 \\
& \text { if } i=2 \\
& \text { if } i=3 \\
& \text { if } i=4
\end{aligned}
$$

$$
\begin{aligned}
& \text { if } i=1 \\
& \text { if } i=2 \\
& \text { if } i=3 \\
& \text { if } i=4
\end{aligned}
$$

$\alpha \in\{$ Intersection with Pre - sigals, Regular Intersection, Realistic Roundabout, Optimistic Roundabout $\}$

$$
K P I_{\gamma}^{\alpha}=\left\{\begin{array}{l}
\sum_{t=1}^{4} \theta_{i} K P I_{1}^{\alpha, t} \\
\frac{\sum_{t=1}^{\alpha} \theta_{t} K P I_{1}^{\alpha, t} K P I_{\gamma}^{\alpha, t}}{K P I_{1}^{\alpha}} 30000 \\
\frac{\sum_{t=1}^{4} \theta_{t} K P I_{1}^{\alpha, t} K P I_{\gamma}^{\alpha, t}}{K P I_{1}^{\alpha}} 7,7+C
\end{array}\right.
$$

By applying formula 3 to calculate $K P I_{1}^{\alpha}$, i.e., the total flow of vehicles per day, the registered flow value on each intensity is multiplied by the number of hours of intensity, during a day. The resulting total flow of each intersection is displayed in Table 1.

Table 1: Estimated total flow per day

\begin{tabular}{|c|c|}
\hline Intersection & Total flow of vehicles \\
\hline $\begin{array}{c}\text { Pre-signals } \\
\text { Regular Intersection } \\
\text { Roundabout }\end{array}$ & 32032 \\
\cline { 2 - 2 } & 31167 \\
\hline
\end{tabular}

As can be seen, the intersection with pre-signals was the one to obtain the highest capacity. However, these Table 2.

$$
\begin{array}{r}
\text { if } \gamma=1 \\
\text { if } 2 \leq \gamma \leq 6 \\
\text { if } \gamma=7
\end{array}
$$

differences are not very significant, making a total difference of about $\mathbf{3 \%}, \mathbf{1 0} \%$ and $\mathbf{2 \%}$, when compared to the regular intersection, realistic roundabout and the optimistic roundabout, respectively.

For the remaining KPI, apart from the queue size, its values were obtained by applying the respective $K P I_{\gamma}^{\alpha}$ formula. Since different amounts of vehicles entered the system, depending on the modelled intersection and its capacity of handling the income traffic, it was necessary to divide the sum by the total flow of each intersection and, afterwards, multiply it by an average number, i.e. 3000 vehicles per day. The obtained results can be consulted in 
Table 2: Estimated total KPI results for an average day and year

\begin{tabular}{|c|c|c|c|c|c|c|}
\hline & Intersection & \begin{tabular}{|c|}
$\begin{array}{c}\text { Total time wasted } \\
\text { (min) }\end{array}$ \\
\end{tabular} & $\begin{array}{c}\text { Total fuel } \\
\text { consumed (g) }\end{array}$ & $\begin{array}{c}\text { Toal CO emissions } \\
\text { (g) }\end{array}$ & $\begin{array}{c}\text { Total HC } \\
\text { emissions (g) } \\
\end{array}$ & $\begin{array}{c}\text { Total NOx } \\
\text { emissions (g) } \\
\end{array}$ \\
\hline \multirow{3}{*}{ Average per cars } & \multirow{3}{*}{$\begin{array}{c}\text { Pre-signals } \\
\text { Regular Intersection } \\
\text { Roundabout }\end{array}$} & 2,96 & 4,55 & 0,90 & 0,07 & 0,06 \\
\hline & & 3,95 & 5,84 & 1,10 & 0,10 & 0,07 \\
\hline & & 4,51 & 8,62 & 1,50 & 0,12 & 0,10 \\
\hline \multirow{3}{*}{$\begin{array}{l}\text { Average per day } \\
\text { (30000 card) }\end{array}$} & \multirow{3}{*}{$\begin{array}{c}\text { Pre-signals } \\
\text { Regular Intersection } \\
\text { Roundabout }\end{array}$} & 88878 & 136371 & 26898 & 2178 & 1770 \\
\hline & & 118395 & 175293 & 33099 & 2928 & 2086 \\
\hline & & 135352 & 258464 & 45094 & 3738 & 2891 \\
\hline \multirow{3}{*}{$\begin{array}{c}\text { Average per year } \\
\text { (30 } 000 \text { cars } * 265 \text { days) }\end{array}$} & \multirow{3}{*}{$\begin{array}{c}\text { Pre-signals } \\
\text { Regular Intersection } \\
\text { Roundabout }\end{array}$} & 31996172 & 49093409 & 9683422 & 784201 & 637340 \\
\hline & & 42622281 & 63105351 & 11915519 & 1054015 & 750787 \\
\hline & & 48726746 & 93047098 & 16233849 & 1345779 & 1040776 \\
\hline
\end{tabular}

As the results illustrate, the vehicles on the intersection with pre-signals were able to cross the intersection in less $\mathbf{2 5 \%}$ time in comparison to the regular intersection and less $\mathbf{3 4 \%}$ in comparison to the roundabout. Moreover, in the intersection with pre-signals, each vehicle could save 1 minute, in comparison to the regular intersection and $\mathbf{2}$ minutes, in comparison to the roundabout.

Regarding the total fuel consumed, the data indicates that, in the intersection modelled with pre-signals, all the vehicles consumed approximately less $\mathbf{2 2 \%}$ of fuel, in comparison to the regular intersection, less $47 \%$ in comparison to the roundabout. Considering all vehicles in a year, these results indicate that there would be a consumption difference of approximately less 14 tons and 44 tons of fuel per year, respectively.

To calculate the average space occupied by a queue on each intersection, formula 3 was applied to calculate $K P I_{\frac{\alpha}{\sigma}}^{\alpha}$. In it, after calculating the average queue size on an average day, it is necessary to multiply the queue size by the average space occupied by a vehicle in a queue (Herman, Lam, and Rothery 1971; Bonneson 1992; Messer and Fambro 1977; Zhu 2007) and to sum a constant $C$, - cf. equation 3 , when $\gamma=7$ depending of the intersection being considered, i.e. $\alpha$ (these values were also used in the simulation model). In this sense, 20 meters were summed to the values related to the roundabouts, since it is the radius of the modelled roundabouts and 40 meters to the intersection modelled with pre-signals. The results are indicated in Table 3 .
Table 3: Average space occupied by a queue on each intersection

\begin{tabular}{|c|c|}
\hline Intersection & $\begin{array}{c}\text { Total space } \\
\text { occupied (meters) }\end{array}$ \\
\hline $\begin{array}{c}\text { Pre-signals } \\
\text { Regular Intersection } \\
\text { Roundabout }\end{array}$ & 220,86 \\
\cline { 2 - 2 } & 282,79 \\
\hline
\end{tabular}

As the table illustrates, the intersection with pre-signals obtained a shorter value than the roundabout, even though the difference is not very significant. It is also possible to verify that its implementation resulted on queues more than $\mathbf{6 0}$ meters shorter that the ones of the regular intersection.

To have an idea of the costs that would be involved in each type of intersection a brief cost analysis was made. It should be noted, however, that these values are estimated, not factual, and their only intention is to give a broad idea of what is the price difference. With this in mind and considering the radius of the roundabouts, i.e. 20 meters, the total area occupied by its infrastructure should be around $1260 \mathrm{~m}^{2}$. Assuming the size of the roads is around 10 meters, it totalizes an area difference of around $560 \mathrm{~m}^{2}$. In its turn, considering a cost of $500 €$ per $\mathrm{m}^{2}$ a global saving of around $280000 €$ is achieved. Moreover, assuming a $5 €$ cost per hour of a person that is wasted waiting on traffic queues, and a $1.5 €$ per litre of fuel ( $1 \mathrm{~kg} \approx 11$ of fuel) Table 4 was built.

Table 4: Cost analysis to the introduction of pre-signals in an intersection

\begin{tabular}{|c|c|r|r|}
\hline $\begin{array}{c}\text { Costs (Difference between } \\
\text { intersection with pre-signals } \\
\text { and the other intersections) }\end{array}$ & $\begin{array}{c}\text { Comparing intersection } \\
\text { with pre-signals and: }\end{array}$ & Per day & Per year \\
\hline Extra Time Cost & Regular intersection & $2460 €$ & $885509 €$ \\
\hline Extra Fuel Cost & Regundabout & $3873 €$ & $1394214 €$ \\
\hline Total & Roundabout & $183 €$ & $65931 €$ \\
\hline & Regular intersection & 2518 & 906527 \\
\hline & Roundabout & 4056 & 1460145 \\
\hline
\end{tabular}

As the table illustrates, the greater part of the savings is obtained as a consequence of the time saved by drivers. In its turn, the savings related to the fuel consumptions are always beneficial to the intersection with presignals. Lastly, considering the total savings per year of the intersection with pre-signals compared to the regular intersection and the roundabout, it can be observed that a global saving of more than $\mathbf{1}$ million $€$ per year, summed over all drivers, could be achieved. 
It should be noted that, despite the small cost analysis of the impact of introducing pre-signals, these do not include the budgets to build the alternative infrastructures, such as roundabouts, bridges or tunnels. In addition, roundabouts are characterized by increasing the fuel consumption and emission of vehicles, due to the constant stop and start-up processes, through which vehicles trying to access it incur. Thus, the environmental and emissions are also expected to decrease with the proposed approach, even though the limited achieved benefits do not reveal such benefits.

\section{CONCLUSIONS}

Usually, the solution for most traffic intersection congestion problems consists in building onerous infrastructures e.g.: tunnels or bridges. This paper presents a novel low-cost solution, consisting in using an additional set of traffic-lights situated some meters away from the main ones, working as pre-signals and acting as "launch-pads" for vehicles. To evaluate this new approach, the authors developed a traffic simulation model on Simio. In previous publications (A. Vieira et al. 2014; A. A. C. Vieira et al. 2017) this new approach had already been compared to both regular signalized intersections and roundabouts, albeit considering static traffic intensities. In this paper, all intersections will be compared, considering the traffic intensities during an average day and a brief analysis over assumed costs was establish,

The intersections were compared on average day and year perspectives. It was found that there were no significant differences regarding the maximum flow of vehicles per day, even though the intersections with presignals obtained the best flow values, thus increasing the capacity of the intersection. Concerning the time spent on each intersection, it was found that on the intersection modelled with pre-signals, per day, all of the modelled vehicles spent less $25 \%$ time than in the regular intersection and less $34 \%$ in comparison to the roundabout. Considering the intersection with presignals, these results culminate in an average time saving of around 1 minute per vehicle in comparison to the regular intersection and 2 minutes in comparison to the roundabout. Regarding the fuel consumptions, the vehicles modelled in the intersection with pre-signals consumed approximately less $22 \%$ of fuel, in comparison to the regular intersection and less $47 \%$ of fuel in comparison to the roundabout. These results culminate in a consumption of approximately less 14 tons and 44 tons of fuel per year, respectively. Regarding the average space occupied, it was observed that there were no significant differences between the values recorded of the optimistic roundabout and the intersection with pre-signals. However, the differences registered between the latter and the regular intersection were more than 60 meters. To finalize the study, a small cost analysis was performed by making some cost assumptions. The authors concluded that the area difference between a traffic signalized intersection and a roundabout with 40 meters of diameter, could result in a saving of around $280000 €$.By further considering the savings per driver, per year, in both time and fuel consumed, the authors concluded that more than 1 million $€$, among all drivers, could be saved. Nonetheless, it should be noted that these values do not include the savings that would be obtained by including the budgets to build other infrastructures to improve the intersection, such as bridges or tunnels, as well as the environmental costs associated to the reduction of the emissions, resulted by the reduction of the fuel consumption.

With this paper, the authors believe to have proposed an implementation which, at least, could be pondered by traffic managers, since, the obtained results indicate that it would be possible to considerably increase the performance of a signalized intersection by simply adding pre-signals to its approaches. Whilst building expensive infrastructures in very saturated traffic intersections is still a mandatory option for certain cites, there are cases in which a less expensive option - such as the pre-signals proposed in this paper - could be used. On the other hand, in the author's view, this paper also contributes to the literature in two ways: firstly, it adds a study that uses a general-purpose discrete-event simulation tool to develop an agent-based traffic micro simulation model - as shows in the first section, most use traffic simulation packages; lastly, it adds a novel implementation for pre-signals in traffic intersections as shows in the first section, most implementations for pre-signals can be divided in either giving priority to buses or to left-turn maneuvers.

\section{ACKNOWLEDGMENTS}

This work has been supported by FCT - Fundação para a Ciência e Tecnologia within the Project Scope: UID/CEC/00319/2019.

\section{REFERENCES}

Bonneson, James A. 1992. "Modeling Queued Driver Behavior at Signalized Junctions." Transportation Research Record, 99.

Cai, Zhengyi, Manchu Xiong, Dongfang Ma, and Dianhai Wang. 2016. "Traffic Design and Signal Timing of Staggered Intersections Based on a Sorting Strategy." Advances in Mechanical $\begin{array}{lll}\text { Engineering } 8 & \text { (4): } 168781401664129 .\end{array}$ https://doi.org/10.1177/1687814016641292.

Dias, Luis M. S., Antonio A. C. Vieira, Guilherme A. B. Pereira, and Jose A. Oliveira. 2016. "Discrete Simulation Software Ranking — A Top List of the Worldwide Most Popular and Used Tools." In 2016 Winter Simulation Conference (WSC), 1060-71. IEEE. https://doi.org/10.1109/WSC.2016.7822165.

Guler, Lgin, and Monica Menendez. 2014. "Analytical Formulation and Empirical Evaluation of PreSignals for Bus Priority." Transportation Research Part B: Methodological 64 (June): 4153. https://doi.org/10.1016/j.trb.2014.03.004.

Guler, S. Ilgin, Vikash V. Gayah, and Monica 
Menendez. 2016. "Bus Priority at Signalized Intersections with Single-Lane Approaches: A Novel Pre-Signal Strategy." Transportation Research Part C: Emerging Technologies 63 (February): 51-70. https://doi.org/10.1016/j.trc.2015.12.005.

Guler, S. Ilgin, and Monica Menendez. 2014. "Evaluation of Presignals at Oversaturated Signalized Intersections." Transportation Research Record: Journal of the Transportation Research Board 2418 (1): 11-19. https://doi.org/10.3141/2418-02.

Herman, Robert, T Lam, and R W Rothery. 1971. "The Starting Characteristics of Automobile Platoons." Publication of: Traffic Flow and Transportation.

Hlupic, V, and R J Paul. 1999. "Guidelines for Selection of Manufacturing Simulation Software." IIE Transactions $31 \quad$ (1): 21-29. https://doi.org/10.1023/A:1007568516643.

Hlupic, Vlatka. 2000. "Simulation Software: An Operational Research Society Survey of Academic and Industrial Users." In Winter Simulation Conference Proceedings, 2:1676-83.

Kozey, Peter, Yiguang Xuan, and Michael J. Cassidy. 2016. "A Low-Cost Alternative for Higher Capacities at Four-Way Signalized Intersections." Transportation Research Part C: Emerging Technologies 72 (November): 157-67. https://doi.org/10.1016/j.trc.2016.09.012.

Li, Yan, Ke Li, Siran Tao, Xia Wan, and Kuanmin Chen. 2014. "Optimization of the Design of PreSignal System Using Improved Cellular Automaton." Computational Intelligence and Neuroscience 2014: 1-11. https://doi.org/10.1155/2014/926371.

Messer, Carroll J, and Daniel B Fambro. 1977. Effects of Signal Phasing and Length of Left-Turn Bay on Capacity.

Oakes, J, A. M. Hellmann, and I. T. .Kelly. 1994. "Innovative Bus Priority Measures." In Proceedings of Seminar J, Traffic Management and Road Safety, 22nd PTRC European Transport Summer Annual Meeting, 301-12. Proceedings of Seminar J, Traffic Management and Road Safety, 22nd PTRC European Transport Summer Annual Meeting, University of WARWICK, U.K., vol.381, pp.301-312.

Oueida, Soraia, Pierre Abi Char, Seifeddine Kadry, and Sorin Ionescu. 2016. "Simulation Models for Enhancing the Health Care Systems." FAIMA Business \& Management Journal 4 (4): 5-20. http://search.ebscohost.com/login.aspx?direct=tru $\mathrm{e} \& \mathrm{db}=\mathrm{bsu} \& \mathrm{AN}=121321531 \&$ site=ehost-live.

Pan, Maolin, Sheng Dong, Jian Sun, and Keping Li. 2010. "Microscopic Simulation Research on Signal Cycle Length of Mixed Traffic Considering Violation." In 2010 International Conference on Intelligent Computation Technology and Automation, 674-78. IEEE. https://doi.org/10.1109/ICICTA.2010.658.
Vieira, A., L.S. Dias, G.A.B. Pereira, J.A. Oliveira, M.S. Carvalho, and P. Martins. 2015. "Using Simio to Automatically Create 3d Warehouses and Compare Different Storage Strategies." FME Transactions 43 https://doi.org/10.5937/fmet1504335V.

Vieira, A., L.S. Dias, G.B. Pereira, and J.A. Oliveira. 2014. Micro Simulation to Evaluate the Impact of Introducing Pre-Signals in Traffic Intersections. Lecture Notes in Computer Science (Including Subseries Lecture Notes in Artificial Intelligence and Lecture Notes in Bioinformatics). Vol. 8584 LNCS. https://doi.org/10.1007/978-3-319-091532 254.

Vieira, A.A.C., L.M.S. Dias, G.A.B. Pereira, and J.A. Oliveira. 2017. Agent-Based Simulation to Assess the Performance of Intersections with PreSignals: Comparison with Roundabouts. Lecture Notes in Computer Science (Including Subseries Lecture Notes in Artificial Intelligence and Lecture Notes in Bioinformatics). Vol. 10572 LNCS. https://doi.org/10.1007/978-3-319-68496$3 \_36$.

Vieira, A A C, L M S Dias, G A B Pereira, J A Oliveira, M Do Sameiro Carvalho, and P Martins. 2018. "Simulation Model Generation for Warehouse Management: Case Study to Test Different Storage Strategies." International Journal of Simulation and Process Modelling 13 (4): 32436. https://doi.org/10.1504/IJSPM.2018.093761.

Vieira, António AC, Luís MS Dias, Maribel Y Santos, Guilherme AB Pereira, and José A Oliveira. 2018. "Setting an Industry 4.0 Research and Development Agenda for Simulation - A Literature Review." International Journal of Simulation Modelling 17 (3): 377-90. https://doi.org/10.2507/IJSIMM17(3)429.

Vieira, António, Luís M. S. Dias, Guilherme Pereira, and José A. Oliveira. 2014. "Comparison of SIMIO and ARENA Simulation Tools." In 12th Annual Industrial Simulation Conference (ISC2014), 5-13.

Wu, Jiaming, Pan Liu, Zong Z. Tian, and Chengcheng $\mathrm{Xu}$. 2016. "Operational Analysis of the Contraflow Left-Turn Lane Design at Signalized Intersections in China." Transportation Research Part C: Emerging Technologies 69 (August) 228-41. https://doi.org/10.1016/j.trc.2016.06.011.

Xuan, Yiguang, Carlos F. Daganzo, and Michael J. Cassidy. 2011. "Increasing the Capacity of Signalized Intersections with Separate Left Turn Phases." Transportation Research Part B: Methodological $45 \quad$ (5): $\quad 769-81$. https://doi.org/10.1016/j.trb.2011.02.009.

Yan, Chiwei, Hai Jiang, and Siyang Xie. 2014. "Capacity Optimization of an Isolated Intersection under the Phase Swap Sorting Strategy." Transportation Research Part B: Methodological 60 (February): 85-106. https://doi.org/10.1016/j.trb.2013.12.001. 
Yang, Qiaoli, and Zhongke Shi. 2017. "Performance Analysis of the Phase Swap Sorting Strategy for an Isolated Intersection." Transportation Research Part C: Emerging Technologies 77 (April): 366-88. https://doi.org/10.1016/j.trc.2017.01.018.

Zhao, Jing, and Yue Liu. 2017. "Safety Evaluation of Intersections with Dynamic Use of Exit-Lanes for Left-Turn Using Field Data." Accident Analysis \& Prevention $102 \quad$ (May): 31-40. https://doi.org/10.1016/j.aap.2017.02.023.

Zhao, Jing, Yue Liu, and Di Di. 2016. "Optimization Model for Layout and Signal Design of Full Continuous Flow Intersections." Transportation Letters $\quad 8 \quad$ (4): $\quad 194-204$. https://doi.org/10.1080/19427867.2015.1109752.

Zhao, Jing, Wanjing Ma, K. Larry Head, and Xiaoguang Yang. 2015. "Optimal Operation of Displaced Left-Turn Intersections: A Lane-Based Approach." Transportation Research Part C: Emerging Technologies 61 (December): 29-48. https://doi.org/10.1016/j.trc.2015.10.012.

Zhao, Jing, Wanjing Ma, H. Michael Zhang, and Xiaoguang Yang. 2013. "Increasing the Capacity of Signalized Intersections with Dynamic Use of Exit Lanes for Left-Turn Traffic." Transportation Research Record: Journal of the Transportation Research Board 2355 (1): 49-59. https://doi.org/10.3141/2355-06.

Zhou, Yaping, and Hongbin Zhuang. 2014. "The Optimization of Lane Assignment and Signal Timing at the Tandem Intersection with PreSignal." Journal of Advanced Transportation 48 (4): 362-76. https://doi.org/10.1002/atr.1222.

Zhu, Hong. 2007. "Normal Acceleration Characteristics of the Leading Vehicle in a Queue at Signalized Intersections on Arterial Streets." Master's thesis. Oregon State University.

\section{AUTHORS BIOGRAPHY}

António A.C. Vieira is an Invited Assistant Professor and Researcher of the ALGORITMI Research Centre, University of Minho, Portugal. He holds a MSc degree in Systems Engineering at the University of Minho. He is making his doctoral studies in Advanced Engineering Systems for Industry at the University of Minho, in partnership with Bosch Car Multimedia Braga. His research interests include Modelling and Simulation, Logistics and Transportation, and Big Data. His email address is antonio.vieira@dps.uminho.pt.

Luís M.S. Dias is an Auxiliar Professor and Researcher of the ALGORITMI Research Centre, University of Minho, Portugal. He holds a PhD degree in Production and Systems Engineering from the University of Minho, Portugal. His research interests include Simulation, Systems Performance, Operational Research and Systems Visual Modeling. His email address is 1sd@dps.uminho.pt.
Guilherme A.B. Pereira is an Associate Professor and Researcher of the ALGORITMI Research Centre, University of Minho (UMinho), in Portugal. He holds a $\mathrm{PhD}$ degree in Manufacturing and Mechanical Engineering from the University of Birmingham, UK. His research interests include Operational Research and Simulation. Currently he is Pro-Rector of the University of Minho. His email address is gui@dps.uminho.pt.

José A. Oliveira is an Auxiliar Professor and Researcher of the ALGORITMI Research Centre, University of Minho (UMinho), in Portugal. He holds a PhD degree in Production and Systems Engineering from the University of Minho, Portugal. His research interests include Optimization with Heuristic Methods in Systems Engineering. His email address is zan@dps.uminho.pt. 